\title{
Role of chemokine CX3CL1 in progression of multiple myeloma via CX3CR1 in bone microenvironments
}

\author{
AKINORI WADA ${ }^{1,2^{*}}$, AYA ITO $^{2 *}$, HIROFUMI IITSUKA $^{2}$, KOICHI TSUNEYAMA ${ }^{3}$, \\ TAKAYOSHI MIYAZONO ${ }^{1}$, JUN MURAKAMI ${ }^{1}$, NAOTOSHI SHIBAHARA ${ }^{2}$, HIROAKI SAKURAI ${ }^{3}$, \\ IKUO SAIKI $^{4}$, TAKASHI NAKAYAMA ${ }^{5}$, OSAMU YOSHIE ${ }^{6}$, KEIICHI KOIZUMI $^{2}$ and TOSHIRO SUGIYAMA ${ }^{1}$ \\ ${ }^{1}$ Department of Gastroenterology and Hematology, Graduate School of Medicine and Pharmaceutical Science; \\ ${ }^{2}$ Division of Kampo Diagnostics, Institute of Natural Medicine; ${ }^{3}$ Department of Cancer Cell Biology, \\ Graduate School of Medicine and Pharmaceutical Sciences; ${ }^{4}$ Division of Pathogenic Biochemistry, \\ Institute of Natural Medicine, University of Toyama, Toyama; ${ }^{5}$ Division of Chemotherapy, \\ Kinki University School of Pharmaceutical Sciences; ${ }^{6}$ Department of Microbiology, \\ Kinki University Faculty of Medicine, Osaka, Japan
}

Received February 6, 2015; Accepted March 2, 2015

DOI: $10.3892 /$ or.2015.3941

\begin{abstract}
Several chemokines/chemokine receptors such as CXCL12, CCL3, CXCR4 and CCR1 attract multiple myelomas to specific microenvironments. In the present study, we investigated whether the CX3CL1/CX3CR1 axis is involved in the interaction of the multiple myeloma cells with their microenvironment. The expression of CX3CR1 (also known as fractalkine) was detected in three of the seven human myeloma cell lines. CX3CL1-induced phosphorylation of Akt and ERK1/2 was detected in the CX3CR1-positive cell lines, but not in the CX3CR1-negative cell lines. In addition, CX3CL1-induced cell adhesion to fibronectin and vascular cell adhesion molecule-1 (VCAM-1) in the human myeloma RPMI-8226 cell line. We also investigated whether a relationship existed between myeloma cells and osteoclasts that may function via the CX3CL1/CX3CR1 axis. Conditioned medium from CX3CL1-stimulated RPMI-8226 cells drastically increased the osteoclast differentiation. Collectively, the results from the present study support the concept of the CX3CL1-mediated activation of the progression of the multiple myeloma via CX3CR1. Thus, CX3CR1 may represent a potential therapeutic target for the treatment of multiple myeloma in a bone microenvironment.
\end{abstract}

Correspondence to: Dr Keiichi Koizumi, Division of Kampo Diagnostics, Institute of Natural Medicine, University of Toyama, 2630 Sugitani, Toyama 930-0194, Japan

E-mail: kkoizumi@inm.u-toyama.ac.jp

${ }^{*}$ Contributed equally

Key words: chemokine CX3CL1, survival pathway, adhesion, osteoclast, multiple myeloma

\section{Introduction}

The multiple myeloma cell is a neoplastic plasma-cell disorder that is characterized by clonal proliferation of malignant plasma cells in the bone marrow microenvironment. This disorder causes monoclonal protein proliferation in the blood or urine and it is associated with organ dysfunction (1). It accounts for $\sim 1 \%$ of neoplastic diseases and $\sim 10 \%$ of hematologic cancers. The median age of diagnosis is $\sim 70$ years (2). The recent introduction of autologous stem-cell transplantation and the availability of agents such as thalidomide, lenalidomide and bortezomib have changed the management of myeloma $(3,4)$. Although overall survival has increased, the disease is not curable.

Chemokines are low molecular weight cytokines that are specialized for recruiting leukocyte to inflammatory sites and for correctly positioning lymphocytes in secondary lymphoid organs (5). Chemokines are also involved in different pathological processes including the growth and dissemination of solid tumors and hematological malignancies $(6,7)$. For example, several chemokines/chemokine receptors are associated with multiple myeloma activity. The migration of myeloma cells to the bone marrows is mediated by CXCR4, which is highly expressed in myeloma cells, and by its ligand CXCL12 which is produced by stromal cells (8). CCR1, the receptor for CCL3/MIP-1 $\alpha$, is involved in osteolytic bone diseases and is highly expressed in myeloma patients (9).

CX3CL1 (also known as fractalkine) is a chemokine constitutively expressed in many hematopoietic and non-hematopoietic tissues. It is synthesized as a membranebound protein, but can also be released by proteolytic cleavage $(10,11)$. Membrane-bound CX3CL1 functions as an adhesion molecule, whereas the secreted form triggers chemotaxis of lymphocytes and monocytes to inflammatory sites $(10,12)$. The receptor for CX3CL1, CX3C chemokine receptor 1 (CX3CR1), is expressed on human NK cells, monocytes, $\mathrm{T}$ lymphocytes and mast cells $(10,13)$. 
Previous studies have shown that CX3CR1 expression is upregulated in solid tumors such as in breasts or prostate $(14,15)$, while pancreatic adenocarcinoma models (16) have shown that CX3CR1 is involved in the metastatic spread of tumor cells to specific tissues expressing CX3CL1. Several studies of CX3CR1 have been performed with different types of B cell lymphoma (17) and chronic lymphocytic leukemia (CLL) (18) in hematological malignancies. However, the expression of CX3CR1 has not been investigated in multiple myeloma, since CX3CR1 expression has not been confirmed in B cell lineages from pro B cells to plasma cells (19). Therefore, the functional role of CX3CR1 in multiple myeloma remains unclear. The present study, which was an investigation of the role of the CX3CL1/CX3CR1 axis in multiple myeloma, indicates that this axis is involved in the interaction between the tumor cells and their bone microenvironment.

\section{Materials and methods}

Reagents and antibodies. Recombinant human CX3CL1 and anti-human CX3CL1 were purchased from R\&D Systems (Minneapolis, MN, USA). The following mAbs were used: anti-AKT1 (C-20), anti-ERK1/2 (C-16), anti-PCNA (PC-10) and anti-NFkBp65 (C-20) from Santa Cruz Biotechnology (Santa Cruz, CA, USA), and anti-phospho AKT (Ser-473), anti-phospho p44/42 ERK (Thr-202/Tyr-204), anti-STAT3 and anti-phospho STAT3 (Ser727) from Cell Signaling Technology (Beverly, MA, USA).

Cells. Human multiple myeloma cell lines included RPMI-8226, KMS-12BM, KMS-12PE, L-363, OPM-2, KARPAS-620 and AMO-1 cells. All the cell lines were maintained in RPMI-1640 medium (Wako Pure Chemical Industries, Inc., Osaka, Japan) supplemented with $20 \%$ fetal bovine serum, $50 \mu \mathrm{M}$ 2-mercaptoethanol (both from Invitrogen, Carlsbad, CA, USA), $100 \mathrm{U} / \mathrm{ml}$ penicillin and $100 \mu \mathrm{g} / \mathrm{ml}$ streptomycin (both from Meiji Seika Pharma, Tokyo, Japan). The cells were cultured at $37^{\circ} \mathrm{C}$ in an incubator with a humidified $5 \% \mathrm{CO}_{2}$ atmosphere.

Reverse transcription-polymerase chain reaction (RT-PCR). RNA was extracted using an RNeasy Plus Mini kit (Qiagen, Hilden, Germany) and converted to cDNA using the SuperScript III First-Strand Synthesis System for RT-PCR (Life Technologies Corporation, Carlsbad, CA, USA). The expression of CX3CR1 was determined by RT-PCR. The PCR was performed for 30 cycles (denaturation, $98^{\circ} \mathrm{C} 5 \mathrm{sec}$; annealing, $60^{\circ} \mathrm{C} 5 \mathrm{sec}$; extension, $72^{\circ} \mathrm{C} 10 \mathrm{sec}$ ) using Sapphire Amp Fast PCR Master Mix (Takara Bio, Shiga, Japan) The PCR products were subjected to electrophoresis on a $1.5 \%$ agarose gel with SYBR-Green (Lonza Inc., Rockland, ME, USA) and then photographed under an ultraviolet transilluminator. GAPDH was used as a normalization control. The primers for GAPDH were as follows: sense, 5'-TGA AGG TCG GAG TCA ACG GAT TTG GT-3' and antisense, 5'-CAT GTG GGC CAT GAG GTC CAC CAC-3'. The primers for CX3CR1 were as follows: sense, 5'-TGG CCT TGT CTG ATC TGC TGT TTG-3' and antisense, 5'-ATG GCT TTG GCT TTC TTG TGG TTC-3'. The RNA from healthy donor plasma cell was purchased from AllCells (Alameda, CA, USA).
Western blotting. The western blot analysis was performed following incubation with or without $10 \mathrm{nM}$ rCX3CL1. Antibodies to phosphorylated (p) or unphosphorylated JAK, Stat, Akt and Erk1/2 were used. Images of immunoblots were scanned and quantified with an ImageQuant LAS 4000 Lumino Image Analyzer from Fuji Film Corporation (Tokyo, Japan).

Adhesion assay. Triplicate wells of 96-well plates were coated with $1 \mu \mathrm{g}$ fibronectin (Asahi Techno Glass, Co., Ltd., Tokyo, Japan) and vascular cell adhesion molecule-1 (VCAM-1) (R\&D Systems). RPMI-8226 cells were stimulated by recombinant human CX3CL1 $(10 \mathrm{nM})$ for $5 \mathrm{~min}$. After stimulation, RPMI- 8226 cells $\left(1 \times 10^{4}\right.$ cells $/ 100 \mu 1$ in EMEM with $0.1 \% \mathrm{BSA}$ ) were seeded and incubated for $20 \mathrm{~min}$ at $37^{\circ} \mathrm{C}$. Cells that adhered to the well were evaluated as previously described (20).

Osteoclast differentiation. RPMI-8226 cells were cultured in the absence or presence of recombinant human CX3CL1 $(10 \mathrm{nM})$ for $48 \mathrm{~h}$, and then the conditioned cell culture medium was collected. Osteoclast precursors (RAW 264.7) were suspended in a-MEM supplemented with $10 \%$ FBS and cultured in a 24 -well culture plates at $1 \times 10^{6} /$ well. After $48 \mathrm{~h}$, the culture medium was replaced with $50 \%$ conditioned medium with or without $100 \mathrm{ng} / \mathrm{ml}$ of mouse recombinant soluble RANKL (Wako Pure Chemical Industries, Inc.). After 4 days, the cells were dehydrated with ethanol-acetone (1:1) for $1 \mathrm{~min}$, dried and stained at room temperature with tartrate-resistant acid phosphatase (TRAP) staining solution. TRAP-positive cells appeared dark red. We counted TRAP-positive multinucleated cells containing three or more nuclei as osteoclasts.

Statistical analysis. Data were analyzed for statistical significance using the Student's t-test. $\mathrm{P}<0.05$ was considered to indicate a statistically significant result. The mean and SD were calculated for all variables.

\section{Results}

CX3CR1 expression in multiple myeloma. We first compared the expression of CX3CR1 in seven human multiple myeloma cell lines and plasma cells (CD138-positive cell fraction) by RT-PCR (Fig. 1). CX3CR1 expression was detected in three (RPMI-8226, OPM-2 and KARPAS-620) of the seven human multiple myelomas, even though CX3CR1 was not expressed in the plasma cells that derived from healthy donors. CX3CR1 expression was apparently induced during the process of malignant transformation of normal plasma cells to multiple myeloma.

Activation of Akt and ERK signaling by CX3CL1 in multiple myeloma. The observation of the CX3CR1 expression in human multiple myeloma cells prompted us to examine the biological responses of these cells to CX3CL1. The mechanisms underlying the differences in CX3CL1-induced progression and cell survival were investigated by analyzing signal transduction. Seven multiple myeloma cell lines were incubated with or without recombinant CX3CL1 and were subjected to western blotting using antibodies against unphosphorylated and 


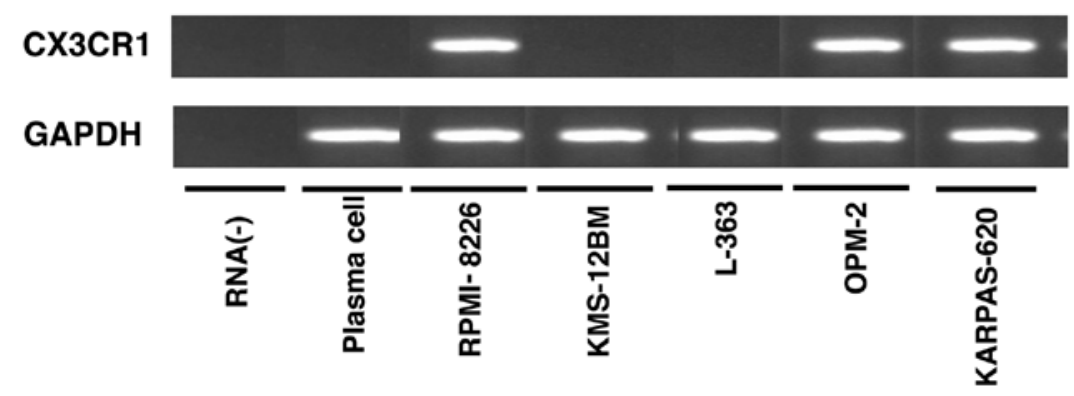

Figure 1. Semi-quantitative RT-PCR analyses of the expression of chemokine receptors CXC3R1 in seven human multiple myeloma cell lines (KMS-12PE, AMO-1, RPMI-8226, KMS-12BM, L-363, OPM-2 and KARPAS620). PHA-treated PBMC was used as a positive control and human plasma cells from healthy donors were negative for CX3CR1 expression. GAPDH at the bottom of the panel served as a loading control. CX3CR1 expression was detected in three cell lines (RPMI-8226, OPM-2 and KARPAS-620). One representative experiment out of three performed is shown.

A

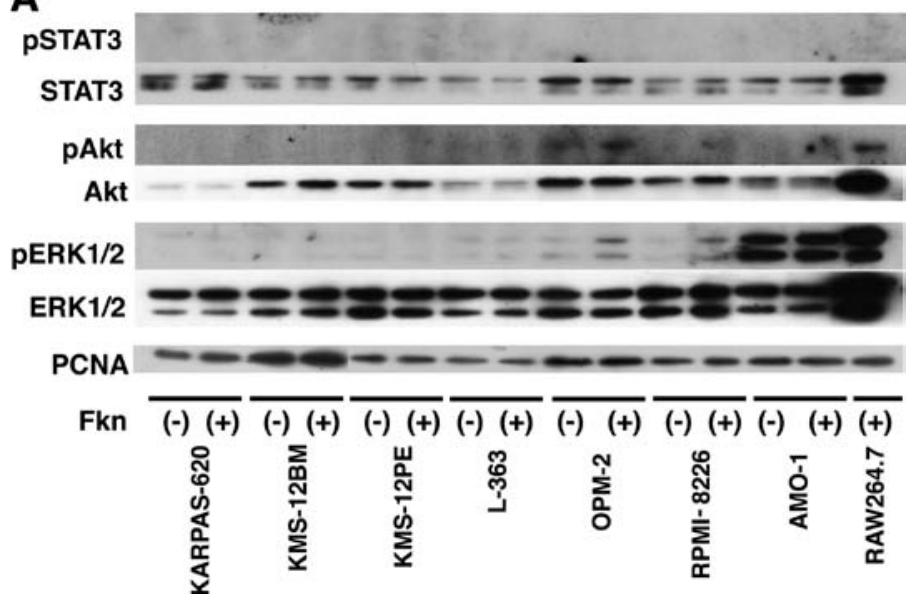

B

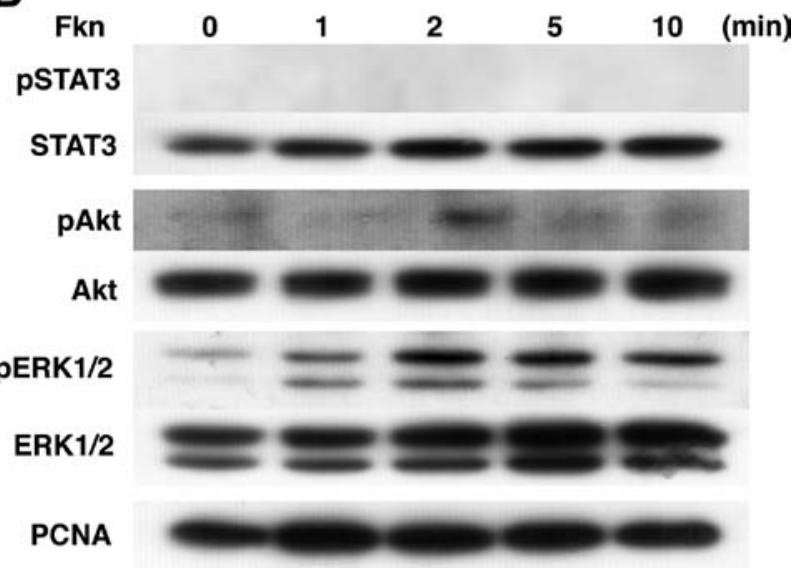

Figure 2. Western blot analysis of Akt, ERK1/2 and STAT3 protein expression and time course of the induction of Akt, ERK1/2 and STAT3 activities by CX3CL1 in (A) seven multiple myeloma cell lines and in (B) RPMI-8226. These cells were treated with CX3CL1 (10 mM, 0-10 min), as indicated. Total Akt, ERK1/2 and STAT3 protein as well as their phosphorylated forms including Akt phosphorylated in serine ( $\mathrm{p}^{\text {Ser473 }}$-Akt) and phosphorylated ERK1/2 ( $\mathrm{p}^{\text {Thr-202/Tyr- }}$ ${ }^{204}$-ERK1/2) and phosphorylated STAT3 ( $\mathrm{p}^{\text {Ser727}}$-STAT3) were detected in the whole cell lysates. The accumulation of phosphorylated Akt and ERK1/2 was strongly and rapidly induced by CX3CL1 in RPMI-8226. No changes were observed in the total protein levels. One representative experiment out of three performed is shown.

phosphorylated Akt, Erk1, Erk2 and STAT3. Akt, Erk1 and Erk2, but not STAT3, were constitutively phosphorylated in the two cell lines (Fig. 2A). Incubation of RPMI-8226 cells with CX3CL1-induced p-Erk1/2 expression after $1 \mathrm{~min}$; this expression peaked from 2 to $5 \mathrm{~min}$, while p-Akt expression peaked after 2 min (Fig. 2B). Next, the RPMI- 8226 cells were stimulated with the antibody for $2 \mathrm{~min}$. We also observed that CX3CL1-induced activation of Akt and ERK in RPMI-8226 cells was inhibited selectively by an anti-CX3CL1 antibody (Fig. 3). These results indicate a rapid CX3CL1-driven signaling for progression and cell survival after stimulation of CX3CL1 in multiple myeloma.

Increased adhesion to the extracellular matrix (ECM) by CX3CL1 in RPMI-8226. We next examined whether CX3CL1 regulates cell adhesion of human multiple myeloma. The number of RPMI-8226 cells adhering to fibronectin and VCAM-1 increased by $\sim 17$ - and 3-fold respectively, in response to pretreatment with recombinant CX3CL1 (Fig. 4). The adhesion of multiple myeloma cells to fibronectin and VCAM-1, which are mainly expressed in bone marrow stromal cells, activates many pathways and results in the upregulation of the

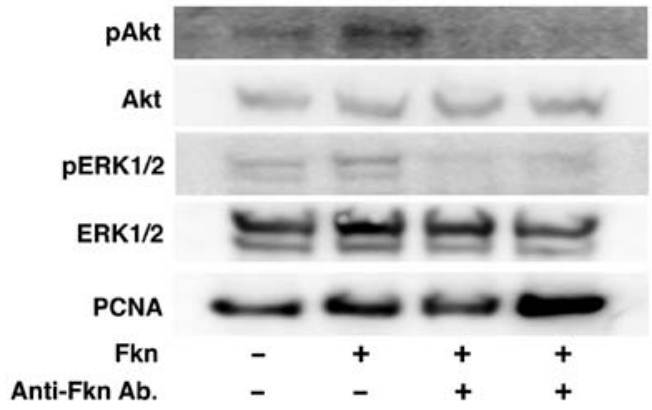

Figure 3. Anti-CX3CL1 antibody $(10 \mu \mathrm{g} / \mathrm{ml})$ was added after initiation of chemokine treatments. Akt and ERK1/2 phosphorylation in response to chemokine at 2 min was assessed in the absence (0) or presence of this antibody. Constitutively phosphorylated Akt and phosphorylated ERK1/2 in RPMI-8226 were inhibited even at low concentrations of anti-CX3CL1 antibody $(10 \mu \mathrm{g} / \mathrm{ml})$, whereas no changes were observed in their total protein expression. PCNA at the bottom of the panel served as a loading control. One representative experiment out of the three performed is shown.

cell cycle regulating proteins and anti-apoptotic proteins $(9,21)$. These results suggest that CX3CL1-induced the progression of multiple myeloma in bone microenvironments. 




Figure 4. CX3CL1 induces adhesion of multiple myeloma cells. RPMI-8226 was stimulated by recombinant human CX3CL1 $(10 \mathrm{nM})$ for $5 \mathrm{~min}$. The cells were added to wells that were pre-coated with fibronectin and VCAM-1 for $20 \mathrm{~min}$ and then washed. After stimulation, the RPMI-8226 cells were seeded and incubated for $20 \mathrm{~min}$ at $37^{\circ} \mathrm{C}$. The cells that adhered to the well were evaluated (pico green). Anti-CX3CL1 antibody $(10 \mu \mathrm{g} / \mathrm{ml})$ was used to ascertain the binding specificity. Data represent the mean $\pm \mathrm{SD}$. ${ }^{*} \mathrm{P}<0.05$. One representative experiment out of the three performed is shown. VCAM-1, vascular cell adhesion molecule-1.

Induction of osteoclast differentiation by multiple myeloma via CX3CL1. We previously reported that CX3CL1 expressed by osteoblasts plays an important role in osteoclast differentiation, possibly acting through its dual functions as a chemotactic factor and adhesion molecule for osteoclast precursors expressing CX3CR1 $(22,23)$.

Given the apparent expression of CX3CR1 by multiple myeloma cells, along with the inducible effect of its ligand CX3CL1 on multiple myeloma cell adhesion to bone microenvironment ECM. We also investigated the possible synergy between multiple myeloma and osteoclast precursors in osteoclast differentiation via CX3CL1. Osteoclast precursors, RAW 264.7 were differentiated by RANKL in conditioned medium collected from multiple myeloma cells stimulated by CX3CL1. After 4 days, TRAP-positive multinuclear osteoclasts were counted (Fig. 5). The conditioned medium increased the number of TRAP-positive multinuclear osteoclasts, while treatment with rat anti-CX3CL1 mAb suppressed the induction of TRAP-positive multinuclear osteoclasts. These results suggest that CX3CL1 indirectly induces osteoclast differentiation by promoting the secretion of a factor from multiple myeloma in bone microenvironments.

\section{Discussion}

In the development of multiple myeloma, several cytokines, such as IL-6, IGF-1, VEGF and TNF- $\alpha$ directly promote cell survival and angiogenesis. JAK-STAT and IL-6 in particular, are believed to play a central role in cell survival and disease progression in multiple myeloma (24). Each chemokine and its receptor forms an axis that promotes cancer progression via effects on cell survival and angiogenesis (25). The role of the CX3CL1/CX3CR1 axis in the interaction between tumor cells and their microenvironment has been examined in non-Hodgkin lymphoma (17) and CLL (18), but not in multiple myeloma. In the present study, we confirmed expression of the chemokine receptor CX3CR1 in the multiple myeloma cell lines. No CX3CR1 was expressed in the plasma cells that derived from healthy donors (19).

We therefore investigated whether the chemokine CX3CL1 and its ligand CX3CR1 may be associated with cell survival and disease progression. As shown in Figs. 1-3, rapid phosphorylation of Akt and ERK1/2, which is related to signaling for survival and progression, as well as JAK-STAT, was observed following chemokine CX3CL1 treatment. These results may indicate that CX3CR1-positive myeloma cells have advantages regarding survival and progression.

However, the reason why multiple myeloma upregulates CX3CR1, but not in plasma cells from healthy donors, remains unclear. Therefore, further studies are needed to clarify the regulation of CX3CR1 expression by analysis of transcriptional factors (26) and chromosomal translocation related to multiple myeloma progression (27). The CX3CL1/CX3CR1 axis has a known association with several diseases caused by abnormal inflammation, such as rheumatoid arthritis (28). In fact, an animal model of CIA (collagen induced arthritis)



B



Figure 5. Induction of osteoclast differentiation by CX3CL1-pre-stimulating multiple myeloma. (A) The conditioned medium was prepared from RPMI-8226 cells stimulated with recombinant human CX3CL1 (10 nM) for $48 \mathrm{~h}$. The RAW 264.7 cells were cultured with the $100 \mathrm{ng} / \mathrm{ml}$ of mouse recombinant soluble RANKL in the absence of $50 \%$ condition medium (a), in the presence of condition $50 \%$ medium (b), and in the presence of condition $50 \%$ medium and antiCX3CL1 mAb. (B) After 4 days, TRAP-positive multinuclear cells containing three or more nuclei were counted as mature osteoclasts. Data represent the mean $\pm \mathrm{SD}$. ${ }^{*} \mathrm{P}<0.05$. One representative experiment out of the three performed is shown. 
showed a dramatic improvement following the administration of the anti-CX3CL1 antibody. In this case, inflammatory cells permeated into the synovium and bone destruction were controlled (29).

The progression of multiple myeloma requires that the cells adhere to the extracellular matrix components such as fibronectin and VCAM-1 in the bone marrow. Many signaling pathways are activated when multiple myeloma adheres to ECM, resulting in upregulation of cell cycle regulating and anti-apoptotic proteins $(9,21)$. In the present study, we showed that the $\mathrm{CX} 3 \mathrm{CL} 1 / \mathrm{CX} 3 \mathrm{CR} 1$ axis aids in the co-operation between multiple myeloma and osteoclast cells. Treatment with CX3CL1-induced adhesion of multiple myeloma cells to bone ECM and also induced osteoclast differentiation by a secretion factor produced by multiple myeloma cells (Figs. 4 and 5). We previously reported that the CX3CL1/CX3CR1 axis also plays an important role in osteoclast differentiation. Osteoclast precursors selectively expressed CX3CR1, whereas CX3CL1 was expressed by osteoblasts $(22,23)$. The demonstration of CX3CR1 expression in multiple myeloma and CX3CL1 in osteoclast precursors observed in the previous, and the present study strongly indicates that the CX3CL1/CX3CR 1 axis may be an attractive therapeutic target for prevention of the progression of multiple myeloma in bone microenvironments. Future studies should be aimed at investigating the blocking of this axis as a means of inhibiting myeloma progression, as well as suppressing the adverse skeletal-related events common in multiple myeloma.

\section{Acknowledgements}

We would like to thank Ms. Toyomi Kozawa and Mr. Yoshihiro Kuwabara for their technical assistance. The present study was supported by a Grant-in-Aid for Scientific Research (C) (no. 22501042).

\section{References}

1. Kyle RA and Rajkumar SV: Multiple myeloma. N Engl J Med 351: 1860-1873, 2004

2. Kristinsson SY, Landgren O, Dickman PW, Derolf AR and Björkholm M: Patterns of survival in multiple myeloma: A population-based study of patients diagnosed in Sweden from 1973 to 2003. J Clin Oncol 25: 1993-1999, 2007.

3. Brenner H, Gondos A and Pulte D: Recent major improvement in long-term survival of younger patients with multiple myeloma. Blood 111: 2521-2526, 2008.

4. Kumar SK, Rajkumar SV, Dispenzieri A, Lacy MQ, Hayman SR, Buadi FK, Zeldenrust SR, Dingli D, Russell SJ, Lust JA, et al: Improved survival in multiple myeloma and the impact of novel therapies. Blood 111: 2516-2520, 2008.

5. Baggiolini M: Chemokines and leukocyte traffic. Nature 392: $565-568,1998$

6. Raffaghello L, Cocco C, Corrias MV, Airoldi I and Pistoia V: Chemokines in neuroectodermal tumour progression and metastasis. Semin Cancer Biol 19: 97-102, 2009.

7. Pistoia V, Corcione A, Dallegri F and Ottonello L: Lymphoproliferative disorders and chemokines. Curr Drug Targets 7 81-90, 2006

8. Trentin L, Miorin M, Facco M, Baesso I, Carraro S, Cabrelle A, Maschio N, Bortoli M, Binotto G, Piazza F, et al: Multiple myeloma plasma cells show different chemokine receptor profiles at sites of disease activity. Br J Haematol 138: 594-602, 2007.

9. Vallet S, Pozzi S, Patel K, Vaghela N, Fulciniti MT, Veiby P, Hideshima T, Santo L, Cirstea D, Scadden DT, et al: A novel role for CCL3 (MIP-1 $\alpha$ ) in myeloma-induced bone disease via osteocalcin downregulation and inhibition of osteoblast function. Leukemia 25: 1174-1181, 2011.
10. Bazan JF, Bacon KB, Hardiman G, Wang W, Soo K, Rossi D, Greaves DR, Zlotnik A and Schall TJ: A new class of membranebound chemokine with a $\mathrm{CX}_{3} \mathrm{C}$ motif. Nature 385: 640-644, 1997.

11. Umehara H, Bloom ET, Okazaki T, Nagano Y, Yoshie O and Imai T: Fractalkine in vascular biology: From basic research to clinical disease. Arterioscler Thromb Vasc Biol 24: 34-40, 2004.

12. Fong AM, Robinson LA, Steeber DA, Tedder TF, Yoshie O, Imai $\mathrm{T}$ and Patel DD: Fractalkine and $\mathrm{CX}_{3} \mathrm{CR} 1$ mediate a novel mechanism of leukocyte capture, firm adhesion, and activation under physiologic flow. J Exp Med 188: 1413-1419, 1998.

13. Imai T, Hieshima $K$, Haskell $C$, Baba $M$, Nagira $M$, Nishimura M, Kakizaki M, Takagi S, Nomiyama H, Schall TJ, et al: Identification and molecular characterization of fractalkine receptor $\mathrm{CX}_{3} \mathrm{CR} 1$, which mediates both leukocyte migration and adhesion. Cell 91: 521-530, 1997.

14. Shulby SA, Dolloff NG, Stearns ME, Meucci O and Fatatis A: CX3CR1-fractalkine expression regulates cellular mechanisms involved in adhesion, migration, and survival of human prostate cancer cells. Cancer Res 64: 4693-4698, 2004.

15. Andre F, Cabioglu N, Assi H, Sabourin JC, Delaloge S, Sahin A, Broglio K, Spano JP, Combadiere C, Bucana C, et al: Expression of chemokine receptors predicts the site of metastatic relapse in patients with axillary node positive primary breast cancer. Ann Oncol 17: 945-951, 2006.

16. Marchesi F, Piemonti L, Fedele G, Destro A, Roncalli M, Albarello L, Doglioni C, Anselmo A, Doni A, Bianchi P, et al: The chemokine receptor CX3CR1 is involved in the neural tropism and malignant behavior of pancreatic ductal adenocarcinoma. Cancer Res 68: 9060-9069, 2008.

17. Andréasson U, Ek S, Merz H, Rosenquist R, Andersen N, Jerkeman M, Dictor M and Borrebaeck CA: B cell lymphomas express $\mathrm{CX}_{3} \mathrm{CR} 1$ a non-B cell lineage adhesion molecule. Cancer Lett 259: 138-145, 2008.

18. Ferrer A, Ollila J, Tobin G, Nagy B, Thunberg U, Aalto Y, Vihinen M, Vilpo J, Rosenquist R and Knuutila S: Different gene expression in immunoglobulin-mutated and immunoglobulin-unmutated forms of chronic lymphocytic leukemia. Cancer Genet Cytogenet 153: 69-72, 2004.

19. Nakayama T, Hieshima K, Izawa D, Tatsumi Y, Kanamaru A and Yoshie O: Cutting edge: Profile of chemokine receptor expression on human plasma cells accounts for their efficient recruitment to target tissues. J Immunol 170: 1136-1140, 2003.

20. Hojo S, Koizumi K, Tsuneyama K, Arita Y, Cui Z, Shinohara K, Minami T, Hashimoto I, Nakayama T, Sakurai H, et al: High-level expression of chemokine CXCL16 by tumor cells correlates with a good prognosis and increased tumor-infiltrating lymphocytes in colorectal cancer. Cancer Res 67: 4725-4731, 2007.

21. Manier S, Sacco A, Leleu X, Ghobrial IM and Roccaro AM: Bone marrow microenvironment in multiple myeloma progression. J Biomed Biotechnol 2012: 157496, 2012.

22. Saitoh Y, Koizumi K, Sakurai H, Minami T and Saiki I: RANKL-induced down-regulation of $C X 3 C R 1$ via PI3K/Akt signaling pathway suppresses Fractalkine/CX3CL1-induced cellular responses in RAW264.7 cells. Biochem Biophys Res Commun 364: 417-422, 2007.

23. Koizumi K, Saitoh Y, Minami T, Takeno N, Tsuneyama K, Miyahara T, Nakayama T, Sakurai H, Takano Y, Nishimura M, et al: Role of CX3CL1/fractalkine in osteoclast differentiation and bone resorption. J Immunol 183: 7825-7831, 2009.

24. International Myeloma Working Group: Criteria for the classification of monoclonal gammopathies, multiple myeloma and related disorders: A report of the International Myeloma Working Group. Br J Haematol 121: 749-757, 2003.

25. Durie BG and Salmon SE: A clinical staging system for multiple myeloma. Correlation of measured myeloma cell mass with presenting clinical features, response to treatment, and survival. Cancer 36: 842-854, 1975.

26. Eychène $\mathrm{A}$, Rocques $\mathrm{N}$ and Pouponnot $\mathrm{C}$ : $\mathrm{A}$ new MAFia in cancer. Nat Rev Cancer 8: 683-693, 2008.

27. Morito N, Yoh K, Maeda A, Nakano T, Fujita A, Kusakabe M, Hamada M, Kudo T, Yamagata K and Takahashi S: A novel transgenic mouse model of the human multiple myeloma chromosomal translocation $\mathrm{t}(14 ; 16)(\mathrm{q} 32 ; \mathrm{q} 23)$. Cancer Res 71 : 339-348, 2011.

28. Murphy G, Caplice N and Molloy M: Fractalkine in rheumatoid arthritis: A review to date. Rheumatology 47: 1446-1451, 2008.

29. Nanki T, Urasaki Y, Imai T, Nishimura M, Muramoto K, Kubota $\mathrm{T}$ and Miyasaka N: Inhibition of fractalkine ameliorates murine collagen-induced arthritis. J Immunol 173: 7010-7016, 2004. 